\title{
Study on Conceptual Blending Theory in a Multimodal Pro-environmental TV Advertisement
}

\author{
Yue Guan \\ College English Teaching Center, Nanfang College of Sun Yat-Sen University, Guangzhou, China \\ E-mail:guanyue8011@126.com
}

\begin{abstract}
Keywords: Multimodal Pro-environmental TV Advertisement, Conceptual Blending Theory

Abstract: Nowadays, environmental problems have become increasingly prominent. As an effective way to publicize and raise the importance of public awareness of environmental protection, multimodal TV public service advertisements have attracted more and more attention of the whole society. This paper analyses one multimodal pro-environmental TV public service advertisements chosen from "National Excellent Radio and Television Public Service Library" on CNTV based on conceptual blending theory proposed by Fauconnier and Turner. It is found that the cross-space mappings between the input spaces mainly present cause-effect relationship in this multimodal pro-environmental TV advertisement. Meanwhile, image, colour, sound, movement and other communicational modes make much difference to emergent structure in blended space. The study shows that the conceptual blending theory has a good guiding role in creating and making multimodal pro-environmental TV advertisements, and it is conducive to the extensive spread of environmental protection ideas.
\end{abstract}

\section{Introduction}

Environmental issues have long been a global problem. Therefore, how to raise public awareness of environmental protection has become a hot topic attracting more and more attention in the world. As a part of public service advertisements, environmental TV advertisements are an important promotion medium for disseminating ecological and environmental knowledge and raising ecocivilization. Multimodal discourse extends the study of language and focuses on how meaning and function are made in combination with the use of multiple modes of communication, such as visual images, scientific symbolism, gesture, action, music and sound (O'Halloran 2004). It can be seen that environmental TV advertising is a typical dynamic multimodal discourse integrating multiple symbolic modes to achieve the overall meaning of the text. At present, domestic and foreign scholars have studied environmental advertisements from different angles. Their research focuses on systemic functional linguistics, rhetoric, sociology and other fields developed by Bator and Cialdin (2000), Hartmann (2010), Searles (2014), Zhang (2012), Peng (2015), etc., but there are relatively few studies on environmental TV advertisements from the perspective of conceptual blending theory in cognitive linguistics.This paper analyses one multimodal pro-environmental TV public service advertisements (PEPSA) chosen from "National Excellent Radio and Television Public Service 
Library” on CNTV based on conceptual blending theory proposed by Fauconnier and Turner in detail.

\section{Theoretical frame}

Conceptual blending theory was proposed and refined by linguists Fauconnier (1997) and Fauconnier and Turner (1998, 2002). Conceptual blending theory is a cognitive process in which people carry out creative thinking and activities, and the whole process of its occurrence is dynamic (Sun Yi 2013:73). Conceptual blending theory model consists of four mental spaces: input space 1, input space2, generic space, and blended space (Figure1).

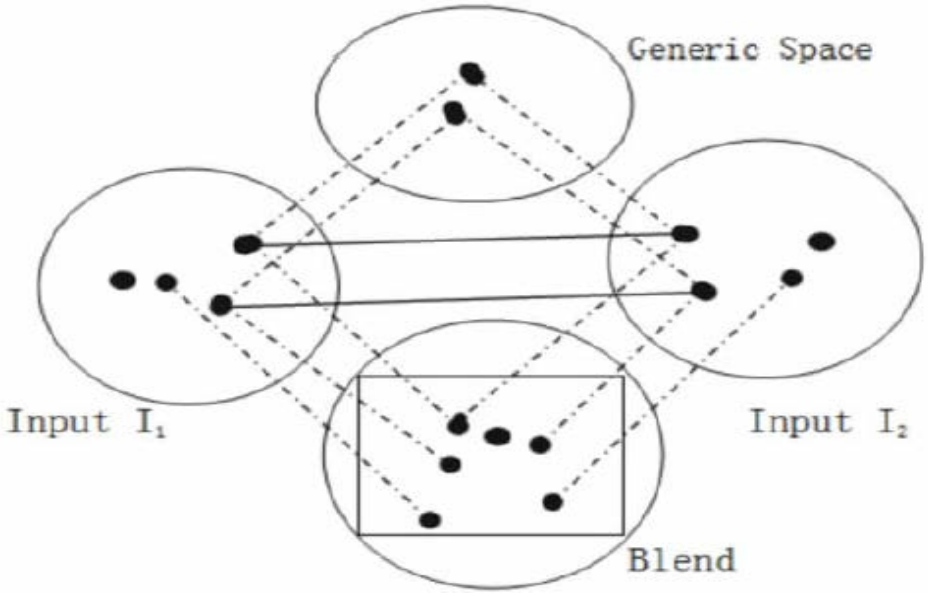

Figure 1. Mental spaces in conceptual blending theory

Input space 1 and Input space 2 are connected by cross-space mapping. The generic mental space maps onto each of the inputs and contains what input spaces have in common. The blended space includes a specific structure of selective projection in two input spaces and an emergent structure established by combining and merging elements. The structure appears as a new, dynamically presented conceptual structure. It can be seen that conceptual blending is a process in which two input spaces are selectively mapped to the blended space through cross-space mapping, and finally a new concept category is generated.

The mapping from input space to blended space includes three basic processes: one is composition, that is, the process of input space projection into the blended space; the second is completion, because some information in the input space is mentioned but not complete; the third is elaboration, which elaborates on the events formed by the integration. The input space maps onto the blended space, largely applying the two processes of combination and refinement. Ungerer and Schmid (2008) and Fauconnier and Turner (2002) pointed out that the process of mapping input space onto blended space is called "compression", which is the process of simplifying the complex concept of multiple input spaces into a new, complete and unified conceptual structure. Compression is the ultimate goal of the entire integration process (Ungerer \& Schmid 2008: 260). There is a cross-space mapping between the two input spaces, which is based on vital relationships between the input spaces including spatial relationships, time relationships, partial-total relationships, cause-effect relationships, event relationships, and so on.

\section{Analytical methods}

Environmental problems are increasingly attracting more and more attention from all over the world. PEPSA play an important component in the "National Excellent Radio and Television Public 
Service Library" on CNTV. Zhao (2013) pointed out that the construction of metaphorical meaning is not limited to the one-way mapping from the source domain to the target domain, but also involves multiple layers of interaction in the discourses such as advertisements. It is believed that the conceptual blending theory is conducive to make up for the multimodal metaphor, which can more clearly explain the metaphor of multimodal construction in emergent structure of the blended space and create new meaning completely. Feng (2011) divided the mapping representations of multimodal metaphors embodied in the texts into three categories: cross-modal mapping, singlemodal mapping and multimodal mapping. Lu Yan (2017), on the basis of Feng Dezheng's research, divided the cross-modal mapping representations into six categories, the single-modal mapping representations into three categories, and the multimodal mapping representations into seven categories. The above-mentioned classifications are not repeated here. In this paper, according to the characteristics of dynamic multimodal discourse of environmental TV advertisements, the labels of verbal (V), pictorial (P), sound/voice (SV), movement (M), colour (C), verbal and pictorial (VP), sound and pictorial (SP), movement and pictorial (MP),colour and pictorial (CP) representation are added. The main purpose of this paper is how to create emergent structure and achieve new meanings through multimodal metaphor and multimodal metonymy as well as the spatial mapping of vital relations effectively in one pro-environmental TV public service advertisement.

\section{Case study}

The pro-environmental TV public service advertisement selected from "National Excellent Radio and Television Public Service Library" titled "Protecting Forests - Hourglass" lasts only 25 seconds. At the beginning of the environmental advertisement, along with the sound mode of the clock ticking, the countdown of the number from 20 to 0 appears, and the sound (characteristic) of the source domain clock activates the target domain time (category). The source domain is characterized by image mode and sound mode, while the target domain is multimodal mapping $\mathrm{S}$ (SP)-T (VP). The moment of the clock ticking brings the audience into 20 seconds, highlighting the conceptual mapping of time in this cognitive domain and constructing the multimodal metonymy of the PROPERTY FOR CATEGORY feature generation category. Later, a beautiful picture of blue sky and white clouds, clear water and green mountains, green trees, singing birds and fragrant flowers appears. The source domain of green trees, birds, blue sky and white clouds refers to the beautiful natural environment. This entity is referred by the source concept, which embodies the referential function of metonymy, forms the source domain represented by image and sound modes, and the target domain is multimodal mapping of image mode $\mathrm{S}(\mathrm{SP})-\mathrm{T}(\mathrm{P})$, constructing a multimodal metonymy PROPERTY FOR CATEGORY. With the time elapsing second by second, following scenes are image modes of haze, hail and other harsh climates, large-scale fallen trees, desertification of the land, and disappearance of forests. The climatic features such as smog and acid rain in the source domain activate the target domain — - the damaged environment (category) through image mode and sound mode. The multimodal mapping of S (SP)-T (VP) embodies the multimodal metonymy PROPERTY FOR CATEGORY.

Ungerer and Schmid (2008) pointed out that generic space is insignificant for understanding conceptual blending theory. Based on Ungerer and Schmid's point of view, this paper only analyzes input space 1, input space 2 and blended space (the same below). The network representation of this TV advertisement integration is shown in Figure 2: 


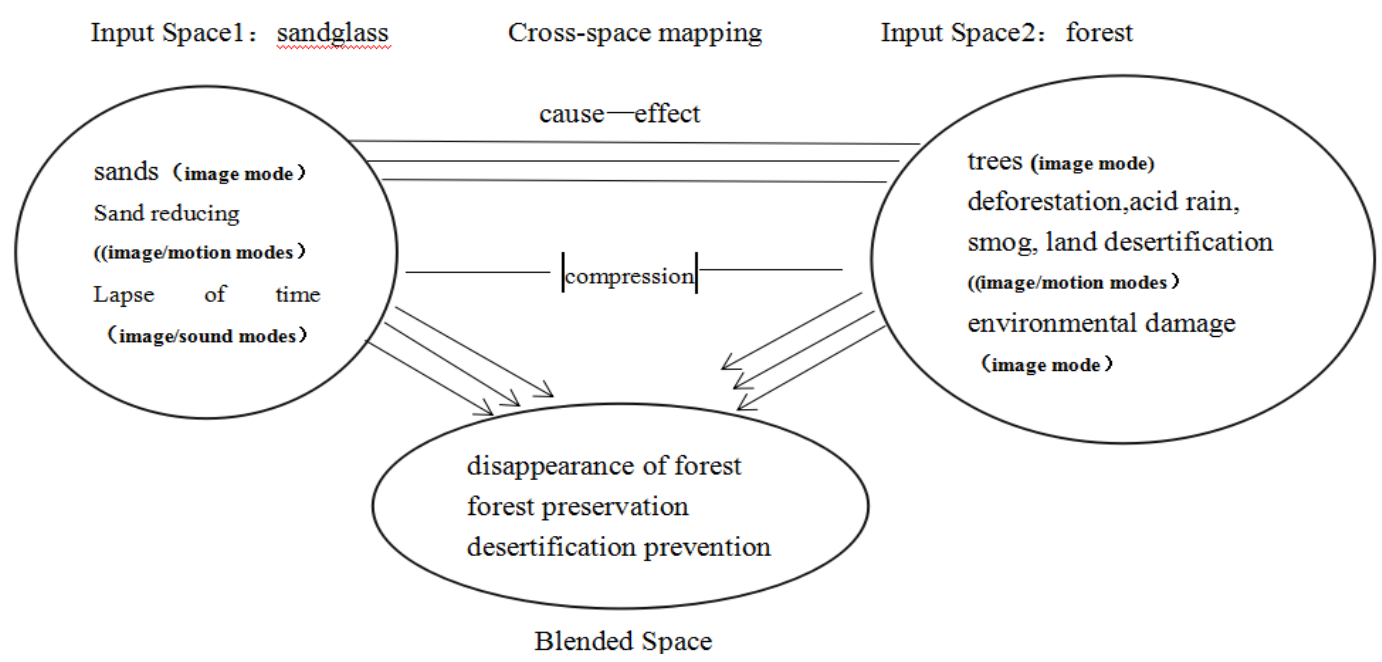

Figure 2. Dynamic construction of conceptual blending theory in "Protection of Forests Hourglass"

As a kind of conceptual metaphor, one of the most typical metaphors is container metaphor, which treats some intangible and abstract events, behaviours, activities and states as concrete, tangible containers. (Sun Yi 2013:68). In the advertisement, the container schema of the source domain of hourglass is mapped onto the target domain of forest by image, sound, text and colour modes, which embodies the source domain as the image mode and the target domain as the multimodal mapping of S (P)-T (VP), constituting multimodal metaphor. Ungerer and Schmid (2008: 127) pointed out that lean mapping between specific source concepts and specific target concepts is mainly used to highlight some specific characteristics of the target concept. Therefore, the mapping between the specific source concept of hourglass and the specific target concept of forest is mainly used to highlight the typical characteristics of the forest crisis and calls for people to protect the forest.

What is the conceptual connection between Input space 1 "hourglass" and Input space 2 "forest”? From Figure 2, the Input space 1 is the image of hourglass provided by the television advertisement. As we all know, the hourglass is a device for measuring time. In this input space, the abstract concept of time is seen as a bounded container. The sand passing through the bounded space (hourglass) creates an intrinsic connection with the time taken from the sand to container. "Forest" in Input space 2 is treated as a tangible container while trees and lands are the containee of this container.

Through the image modes, the source domain — "decreasing sand in the container" in the Input space 1 maps onto Input space 2 “ decreasing numbers of trees and the fertile land in the forest”. It reflects the source domain as the image mode and the target domain as a multimodal mapping $\mathrm{S}$ (P)-T (SP), which constitutes multimodal metaphor.

Through the cross-space mapping, as the measurement time in the hourglass approaches, the fallen trees, deserted land and harsh climate, like the sand in the hourglass, flow into the bottom of the container hourglass. In other words, the dense forest has disappeared (Figure 3). The source domain "sand sinking into the bottom of the hourglass" through the image mode and sound mode is mapped onto the target domain "forest disappearance and land desertification", which embodies multimodal mapping with the source domain as the image and the sound modes, and the target domain as S(SP)-T(VP), constituting multimodal metaphor. The multimodal mapping of the state S (SP)-T (VP) constitutes a multimodal metaphor.

By blending input space 1 and input space 2, the reduction of sand in the hourglass and the lapse of time form a cause-effect relationship; the deforestation of a large number of trees in the forest 
and the desertification of the land represents a cause-result relationship with the disappearance of the forest. As a result, it can be seen that cause-result relationship in the input space 1 and the input space 2 is compressed into emergent structure in the blended space by the cross-space mapping.

At the end of this advertisement, we can see that there are 15000 square meters of forests disappearing in 20 seconds through the language mode. The time of 20 seconds and the disappearance of the forest, interpreted as cause-result relationship, are once again perfectly integrated, and its blended meaning is fully presented in the emergent structure. One is to let the audience recognize the serious situation that the forest is disappearing gradually; the other is to let the audience deeply feel the importance and urgency of protecting the forest and preventing desertification. It is imperative to protect the environment.

\section{Conclusion}

This paper analyses one multimodal environmental TV advertisement based on conceptual blending theory. The research finds that: (1) The source and target domains of the input spaces are mainly composed of multimodal metaphors and multimodal metonymy through images, movement, sound, and other communicational modes in this PEPSA. These modes are important resources generated by the emergent structure in the blended space and make much difference to convey the theme of environmental protection. (2) The cross-space mappings between the input spaces mainly present cause-effect relationship in this multimodal pro-environmental TV advertisement. The research shows that the conceptual blending theory has a good guiding role creating and making multimodal pro-environmental TV advertisement, and it is conducive to spread environmental protection ideas extensively.

\section{Acknowledgments}

This paper was financially supported by the major project of "Youth Innovative Talent Project in Guangdong Province (Humanities and Social Sciences)" in 2015 (project number: 2015WQNCX190); “Foreign Language Teaching and Scientific Research Project of Universities in China" in 2017 (project number: 2017GD0023B), the key project of “Teaching Reform Research in Nanfang College of Sun Yat-Sen University” in 2017 (project number: 2017XJG08) and China Scholarship Council.

\section{References}

[1] Bator, R.J. \& Cialdini, R. B. "The Application of Persuasion Theory to the Development of Effective Proenvironmental Public Service Announcements,” Journal of Social Issues, 2000, pp.527-541.

[2] Fauconnier, G, Mappings in Thought and Language. Cambridge University Press, 1997.

[3] Fauconnier, G. \& Turner, M, The Way We Think: Conceptual Blending and the Mind's Hidden Complexities. New York: Basic Books, 2002.

[4] Forceville, C.\&Urios-Aparisi E, Multimodal Metaphor.Berlin: Mouton DeGruyter, 2009.

[5] Ungerer, F. \& Schmid, H. J, An Introduction to Cognitive Linguistics (2nd Edition). Beijing, Foreign Language and Research Press, 2008.

[6] Fu Yin Li, The Introduction to Cognitive Linguistics. Beijing University Press, 2013.

[7] Yi Sun, Cross-domain Study in Cognitive Metaphor. Beijing University Press, 2013.

[8] Zai Jiang Wei, “Conceptual Blending, Pragmatic Reason and Metonymic Cognition,” Foreign Chinese, 2007, pp.90-95.

[9] Hui Zhang, Wei Zhong Lu, Cognitive Metonymy. Shanghai Foreign Education Press 2010.

[10] Xiu Feng Zhao, “Conceptual Blending of multimodal Metaphor Construction-Exemplified by Political Comics,” Foreign Research 2013, pp.1-8. 